\title{
Construction and sequencing of an infectious clone of the goose embryo-adapted Muscovy duck parvovirus vaccine strain FZ91-30
}

\author{
Jianye Wang ${ }^{1 *}$, Yu Huang ${ }^{1}$, Mingxu Zhou ${ }^{1}$, Philip R. Hardwidge ${ }^{2}$ and Guogiang Zhu ${ }^{1 *}$
}

\begin{abstract}
Background: Muscovy duck parvovirus (MDPV) is the etiological agent of Muscovy duckling parvoviral disease, which is characterized by diarrhea, locomotive dysfunction, stunting, and death in young ducklings, and causes substantial economic losses in the Muscovy duck industry worldwide. FZ91-30 is an attenuated vaccine strain that is safe and immunogenic to ducklings, but the genomic information and molecular mechanism underlining the attenuation are not understood.

Methods: The FZ91-30 strain was propagated in 11-day-old embryonated goose eggs, and viral particles were purified from the pooled allantoic fluid by differential centrifugation and ultracentrifugation. Single-stranded genomic DNA was extracted and annealed to form double-stranded DNA. The dsDNA digested with Ncol resulted two sub-genomic fragments, which were then cloned into the modified plasmid pBluescript II SK, respectively, generating plasmid pBSKNL and pBSKNR. The sub-genomic plasmid clones were sequenced and further combined to construct the plasmid pFZ that contained the entire genome of strain FZ91-30. The complete genome sequences of strain FM and $Y Y$ and partial genome sequences of other strains were retrieved from GenBank for sequence comparison. The plasmid pFZ containing the entire genome of FZ91-30 was transfected in 11-day-old embryonated goose eggs via the chorioallantoic membranes route to rescue infectious virus. A genetic marker was introduced into the rescued virus to discriminate from its parental virus.
\end{abstract}

Results: The genome of FZ91-30 consists of 5,131 nucleotides and has $98.9 \%$ similarity to the FM strain. The inverted terminal repeats (ITR) are 456 nucleotides in length, 14 nucleotides longer than that of Goose parvovirus (GPV). The exterior 415 nucleotides of the ITR form a hairpin structure, and the interior 41 nucleotides constitute the D sequence, a reverse complement of the D' sequence at the $3^{\prime}$ ITR. Amino acid sequence alignment of the VP1 proteins between FZ91-30 and five pathogenic MDPV strains revealed that FZ91-30 had five mutations; two in the unique region of the VP1 protein (VP1 1 ) and three in VP3. Sequence alignment of the Rep1 proteins revealed two amino acid alterations for FZ91-30, both of which were conserved for two pathogenic strains YY and P. Transfection of the plasmid pFZ in 11-day-old embryonated goose eggs resulted in generation of infectious virus with similar biological properties as compared with the parental strain.

Conclusions: The amino acid mutations identified in the VP1 and Rep1 protein may contribute to the attenuation of FZ91-30 in Muscovy ducklings. Plasmid transfection in embryonated goose eggs was suitable for rescue of infectious MDPV.

Keywords: Muscovy duck parvovirus, Attenuation, Infectious clone, Rescue, Transfection

\footnotetext{
* Correspondence: wangjy@yzu.edu.cn; yzgqzhu@yzu.edu.cn

'College of Veterinary Medicine, Yangzhou University, 48 Wenhui East Road,

225009 Yangzhou, Peoples' Republic of China

Full list of author information is available at the end of the article
}

(c) 2016 The Author(s). Open Access This article is distributed under the terms of the Creative Commons Attribution 4.0 International License (http://creativecommons.org/licenses/by/4.0/, which permits unrestricted use, distribution, and reproduction in any medium, provided you give appropriate credit to the original author(s) and the source, provide a link to the Creative Commons license, and indicate if changes were made. The Creative Commons Public Domain Dedication waiver (http://creativecommons.org/publicdomain/zero/1.0/) applies to the data made available in this article, unless otherwise stated. 


\section{Background}

Muscovy duck parvovirus (MDPV) belongs to the Dependoparvovirus genus in the Parvoviridae family. Its closest relative is Goose parvovirus (GPV) [1]. MDPV is the etiological agent of Muscovy duckling parvoviral disease [2-5], which is also named "three-week disease" in China [6]. The disease is characterized by diarrhea, locomotive dysfunction, stunting, and death in young ducklings [2,7], and causes substantial economic losses in the Muscovy duck industry worldwide.

The MDPV genome is a linear, single-stranded DNA molecule of $\sim 5.1 \mathrm{~kb}$ [8]. The genome is flanked by inverted terminal repeats (ITR; 457 nts in the FM strain) that can form a palindromic hairpin structure to serve as origin of replication [9]. The ITR also contains cisacting elements required for rescue, excision from cloning vectors, and packaging [10].

The left open reading frame encodes the nonstructural protein Rep1 as well as several smaller proteins generated after splicing [11, 12]. Rep proteins can bind to ITR elements and are involved in genome replication, and modulation of the downstream P41 promoter $[13,14]$. The right open reading frame encodes the capsid proteins VP1, VP2, and VP3 that are generated from the use of different initiation codons and through proteolytic cleavage. VP1, VP2, and VP3 comprise the viral capsid at a ratio of $\sim 1: 1: 8$ [8].

Live vaccines have been utilized in day-old Muscovy ducklings to prevent disease outbreaks. These attenuated vaccines were prepared by serial passages of the parental derivatives in cell cultures or embryonated eggs [15-17]. FZ91 is a virulent isolate obtained from deceased ducklings that died from an outbreak of "three-week" disease in a farm from Fuzhou city, China [18]. FZ91 was passaged 3 times in embryonated Muscovy eggs and in embryonated goose eggs 30 times, resulting in an attenuated virus that was safe and immunogenic to ducklings. To discriminate the vaccine strain from its parental strain FZ91, the attenuated virus is referred to as FZ91-30.

Here we sequenced the FZ91-30 genome and generated an infectious plasmid clone. We found that several amino acid differences between FZ91-30 and virulent isolates may contribute to MDPV attenuation.

\section{Results}

\section{Genome sequence analysis}

We assembled the complete genome of FZ91-30 after sequencing the sub-genomic fragments. The complete genome is comprised of 5,131 nucleotides and has 98.9 \% similarity to the FM strain, with the latter having an additional " $C$ " nucleotide at the 5 ' terminus of the genome. The ITR of FZ91-30 consists of 456 nucleotides, which forms the palindromic structure at the $5^{\prime}$ and 3 ' termini. 370 nucleotides are involved in basepairing in the stem region of the palindrome, and 45 nucleotides constitute the bubble region. The bubble region (45 nts) has two different sequences in the plusstranded genome, named flip and flop, which form reverse complements. In comparison to the FM strain, FZ91-30 had 4 nucleotide changes in the ITR stems, but at base-pairing positions that did not alter the hairpin structure (Fig. 1). The interior of the ITR is a region designated as the D sequence, comprised of 41 nucleotides. The $\mathrm{D}$ sequence at the $5^{\prime}$ ITR reversely complements with the $\mathrm{D}^{\prime}$ sequence at the 3' ITR.

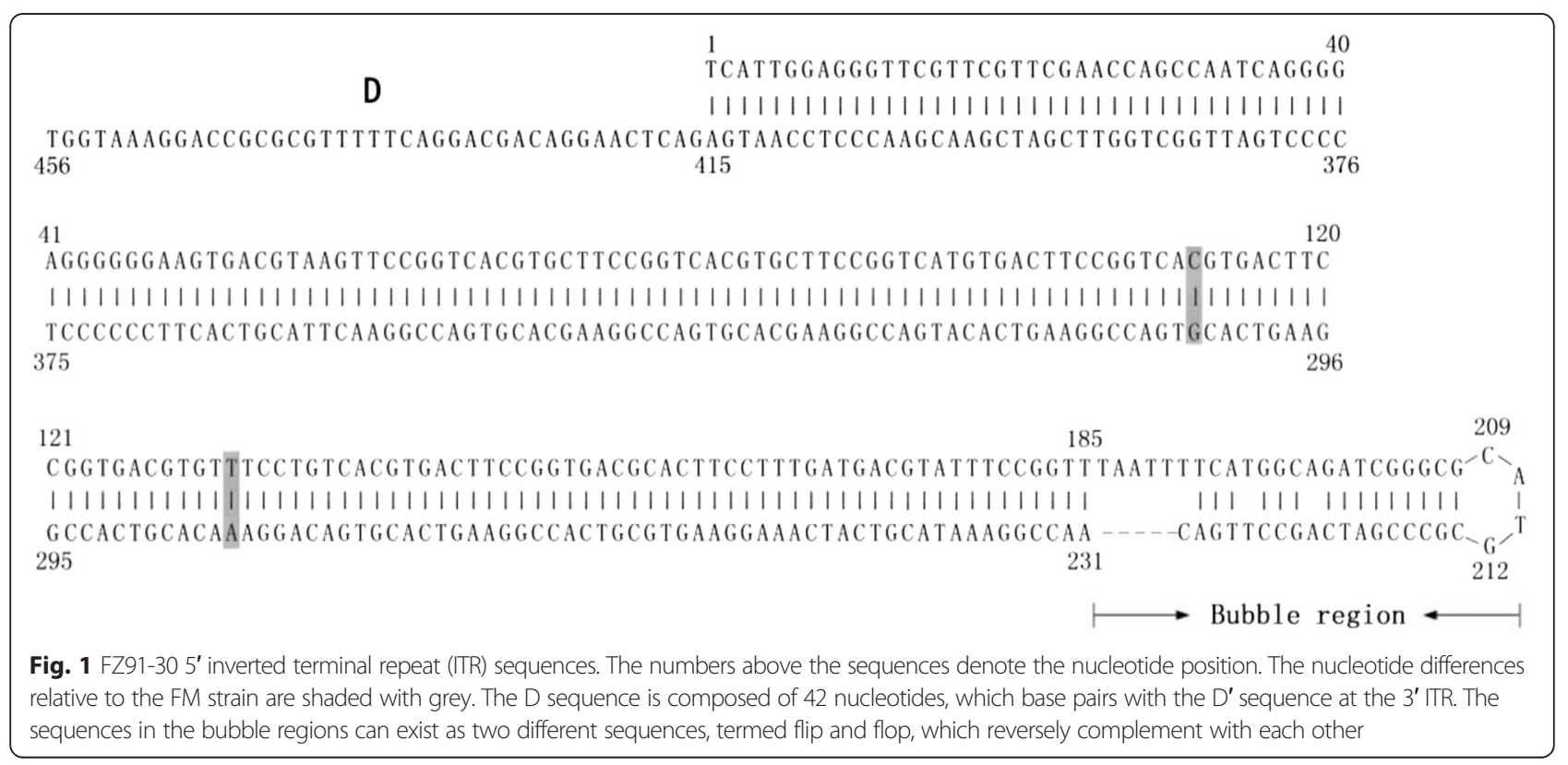




\section{VP1 and Rep1 amino acid sequences comparisons} The VP1 protein of FZ91-30 displayed $98.0 \sim 98.8 \%$ homology at the amino acid level with five pathogenic isolates, $97.8 \%$ homology with the FM strain, and had the highest homology (98.8 \%) with the 89384 strain from France. Amino acid sequence alignments of the VP1 proteins revealed that FZ91-30 had a total of five amino acid differences in comparison to the five pathogenic isolates (89384, 97-0104, 90-0215, 90-0219 and P) (Fig. 2a). Two differences (Q106R and K124R) were in the unique region of the VP1 protein $(\mathrm{VP} 1 \mathrm{u})$, and three variations (V319I, N501K, and P649A) occurred in the VP3 protein. The Rep1 protein of F91-30 displayed $99.5 \%$ and $98.9 \%$ homology at the amino acid level with two pathogenic strain YY and P, respectively. Sequence alignment of the Rep1 proteins revealed that FZ91-30 had produced two amino acid mutations (A223T and F603L), both of which were conserved for strain YY and $\mathrm{P}$ (Fig. 2b). These amino acid differences in VP1 and Rep1 proteins may contribute the attenuation of FZ9130 after serial passages in embryonated goose eggs.

\section{Characterization and rescue of $\mathrm{pFZ}$}

With combination of the cloned sub-genomic fragments, the FZ91-30 genome was cloned into the pBluescript II SK vector (Agilent, Santa Clara, USA), resulting in generation of the plasmid pFZ (Fig. 3a). Since the genome of FZ91-30 lacks BamHI and XhoI sites, digestion of pFZ with BamHI and XhoI produced a 5.1-kb genomic molecule and a $2.9-\mathrm{kb}$ vector molecule. Since there are two SphI sites distributed in the ITRs, digestion of pFZ with $S p h \mathrm{I}$ produced $3.3-\mathrm{kb}$ and $4.7-\mathrm{kb}$ DNA fragments (Fig. 3b).

Embryos began to die 7 days after their transfection with pFZ and mortality rates reached $70 \%$ at day 12 posttransfection. The allantoic fluid was pooled for further passages in 11-day-old embryonated goose eggs. The rescued virus were successfully passaged in embryonated goose eggs four times and killed the embryos between $96 \mathrm{~h}$ and $192 \mathrm{~h}$. The dead embryos showed pathogenic lesions typical of MDPV infection, including hemorrhagic embryos (Fig. 4a) and edematous chorioallantoic membranes. In the control group, all of five goose embryos survived after transfection of the vector plasmid pBSKN during the observation period. Examination of these embryos revealed no obvious pathogenic change (Fig. 4b).

\section{Discrimination of the rescued virus from its parental strain}

A single nucleotide mutation was introduced into the rescued virus. A 1.5-kb DNA fragment covering the mutation site was amplified from the rescued virus. The amplicon from the rescued virus could not be cut with NdeI, while the amplicon from the parental virus could be cut with NdeI, resulting in a $0.7-\mathrm{kb}$ and a $0.8-\mathrm{kb}$ fragment (Fig. 5). The result showed that the rescued virus originated from the transfected plasmid $\mathrm{pFZ}$, eliminating the contamination of the parental virus during viral passages. Sequencing of the whole genome of the rescued virus showed that it had an identical genome sequence to the parental strain FZ91-30, except for the introduced nucleotide mutation in the Rep gene.

\section{Calculation of the $\mathrm{ELD}_{50}$ and MDT}

The medium embryos lethal dose $\left(E L D_{50}\right)$ and mean death time (MDT) of the rescued virus in 11-day-old susceptible embryonated goose eggs was $5 \times 10^{3.5} / \mathrm{ml}$ and $156 \mathrm{~h}$, respectively, while the $\mathrm{ELD}_{50}$ and MDT of the parental strain FZ91-30 was $5 \times 10^{3.36} / \mathrm{ml}$ and $159 \mathrm{~h}$, respectively. Thus the rescued virus had similar viral titers and virulence in embryonated goose eggs as compared with the parental strain.

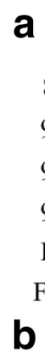

Fig. 2 Amino acid sequence alignments of the VP1 proteins (a) and the Rep1 proteins (b) between the FZ91-30 and other pathogenic isolates. Amino acids in common are indicated by dots. Different amino acids are denoted by the numbers above the alignments and shaded in grey 


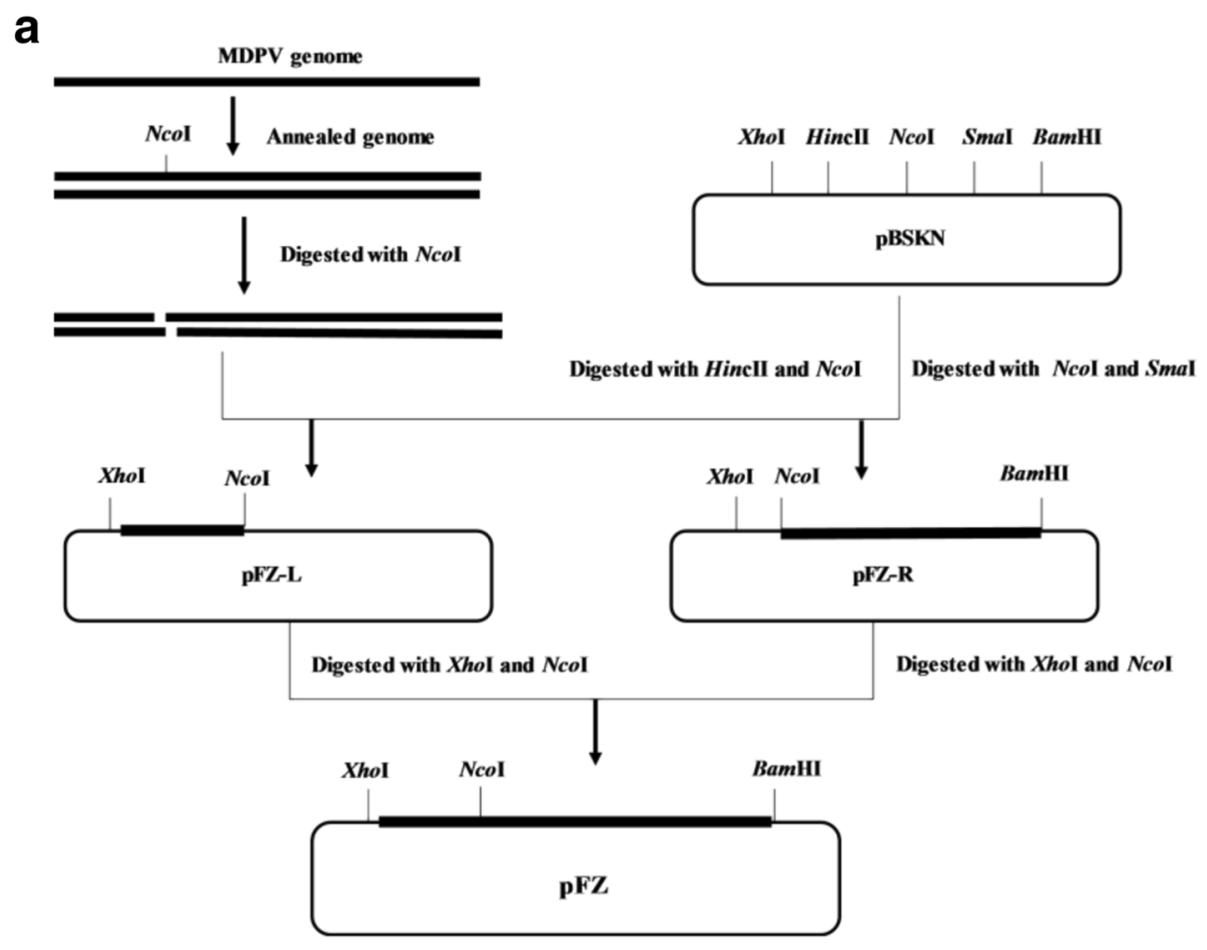

b

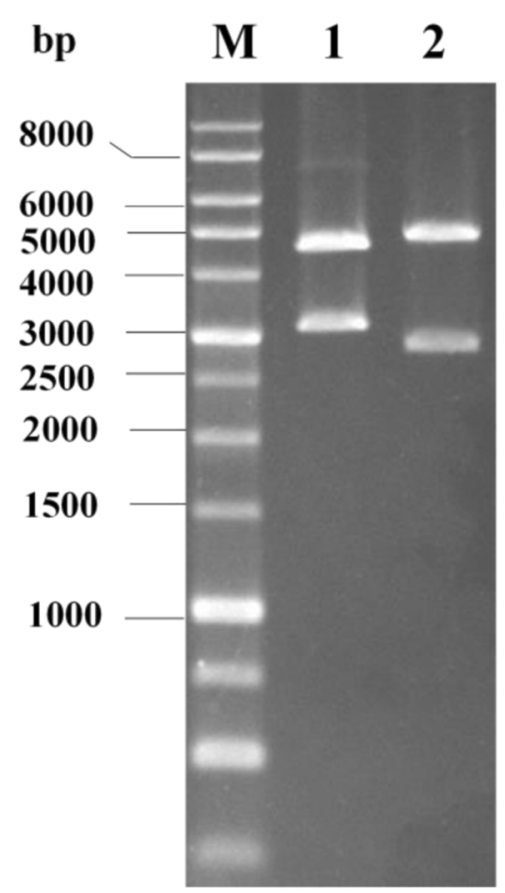

Fig. 3 Experimental strategy used to construct the infectious FZ91-30 MDPV plasmid clone. a The digested MDPV subgenomic fragments were cloned into the pBSKN plasmid respectively and further combined to form the recombinant plasmid pFZ that contained the whole genome of FZ91-30. b Restriction enzyme digestion of pFZ. M: DNA markers. 1: Digestion with Sphl. 2: Double digestion with Xhol and BamHI

\section{Discussion}

Among all the members in the Parvoviridae family, pathogenic waterfowl parvoviruses and the B19 parvovirus possess the longest ITR sequences [9, 19].
Although the genome of MDPV is only 5.1-kb long, the secondary structure in the ITR makes it difficult to clone the full-length genome with one-step PCR amplification or by direct genome cloning $[9,20]$. It is feasible to 

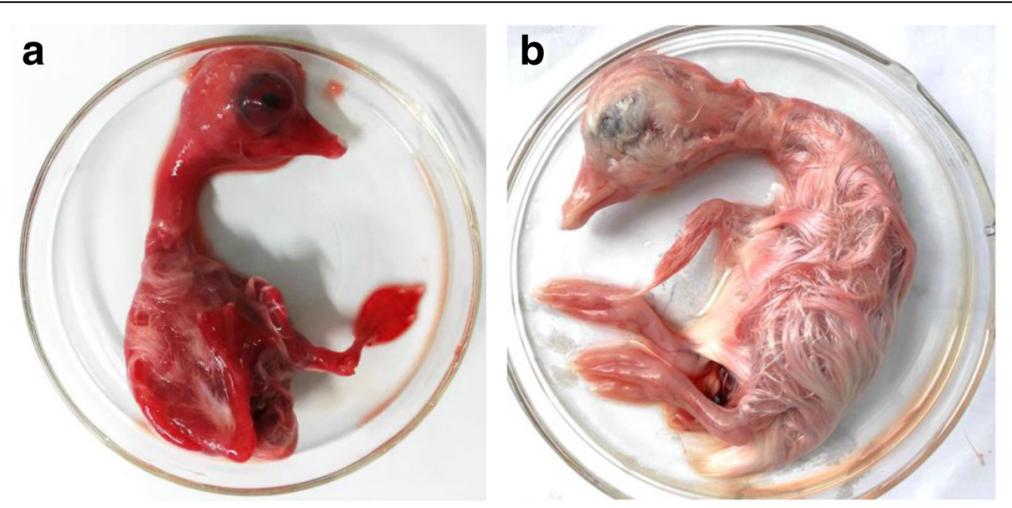

Fig. 4 Pathogenic changes of the embryo died at day 7 post-transfection of pFZ (a), and haemorrhagical lesions in the head, neck, embryonic body, and legs were observed. In the control group (b), no pathogenic change was observed in the embryo which survived in transfection of the vector plasmid pBSKN till the 12th day

clone the sub-genomic fragments respectively and then to combine the sub-genomic fragments to form the fulllength genomic clone. We obtained the plus-stranded genome sequence of MDPV by sequencing several subgenomic clones, except for the sequences in the bubble region at the $5^{\prime}$ terminus and the $3^{\prime}$ terminus. The sequences in the bubble region may exist in two opposite orientations, termed flip and flop, which form reverse complements. The unique SphI site at the loop position of the bubble region allowed us to cut the entire ITR

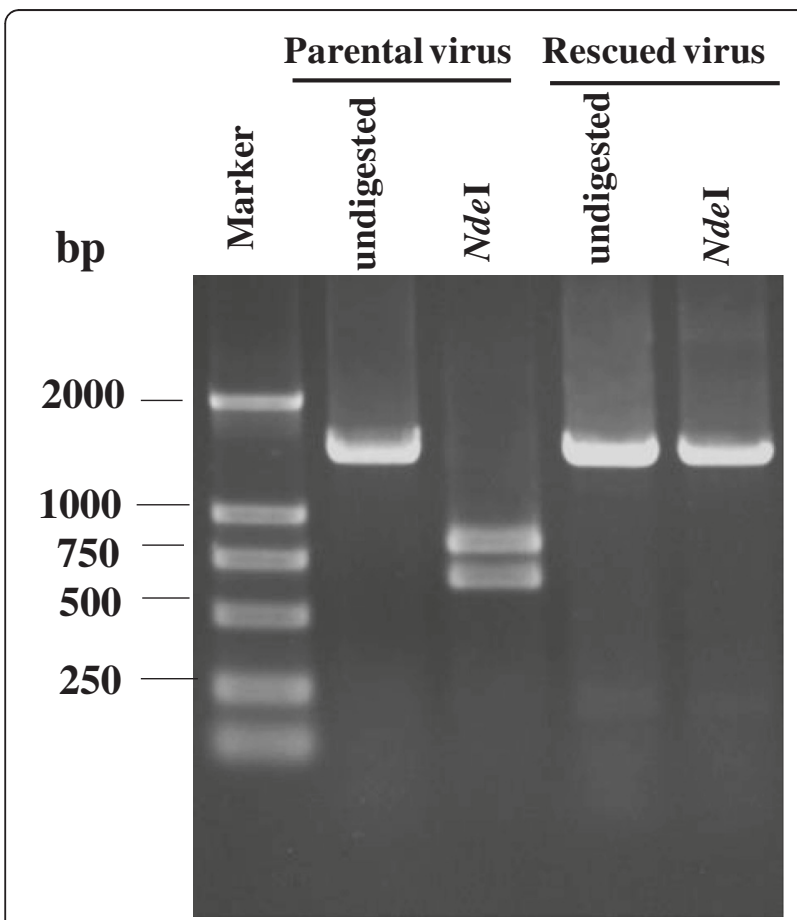

Fig. 5 Discrimination of the rescued virus from the parental strain FZ91-30 using Ndel digestion into two halves and then sequence each subclone containing half of the ITR.

The Sure strain is a kind of E.coli which is engineered for cloning certain DNA segments that are unstable in conventional E. coli strains. The Sure competent cells was used in the transformation experiment for subgenome cloning. Our results herein demonstrated that all of positive plasmid clones from the Sure strain E.coli harbored the ITR sequences without deletions. Simultaneously, we also attempted to use the DH $5 \alpha$ competent cells for transformation comparison with the Sure strain, revealing that all of the screened positive plasmid colonies propagated in DH5 $\alpha$ E.coli contained ITR deletions in various degrees. However, a subset of recombinant plasmids containing the MDPV genome had partially deleted ITRs but were still infectious, as evidenced with successful viral rescue after plasmid transfection in 11day-old embryonated goose eggs. The deleted portions in the ITRs could be repaired in rescued virions by PCR characterization, implying that MDPV might utilize a similar molecular mechanism as adopted by adenoassociated virus (AAV) for correcting its deleted ITRs in the course of viral rescue [21]. The phenomena also supports the current parvoviral classification at some extent that MDPV and GPV are classified into the Dependoparvovirus genus from the primary Autonomous genus [1].

The vaccine strain FZ91-30 is the 30th passage in embryonated goose eggs, which is nonpathogenic for 1-dayold Muscovy ducklings. Prior to our work, complete genome data for MDPV were very limited and most of the MDPV sequences corresponded to the VP1 genes and few sequences about the Rep1 gene were available. Therefore, sufficient VP1 protein sequences from the virulent strains of MDPV could be used for comparison with that of strain FZ91-30 in this study. Six amino acid mutations were identified in the vaccine strain, while these sites were conserved in all five virulent strains of 
MDPV. Parvoviral infection of the host depends on the interaction between the receptor and host cells [22, 23]. The binding domain of the receptor lies in the surface of the viral capsid and thus these mutated amino acids that arose during viral passage probably play a crucial role in the attenuation of FZ91-30. In the previous studies, the phospholipase $\mathrm{A}_{2}\left(\mathrm{PLA}_{2}\right)$ motifs and nuclear localization signals (NLS), which are important for parvoviral infection of host cells, are identified to mainly lie in the VP1 unique region (VP1u) [24, 25]. However, with careful amino acid sequence alignment between FZ91-30 and its counterparts in the Parvoviridae family, we found that both mutations (Q106R and K124R) in the VP1u of FZ91-30 did not reside in the scope of the identified $\mathrm{PLA}_{2}$ and NLS motifs, indicating that the crucial amino acid sites for the activities of PLA $\mathrm{P}_{2}$ and NLS motifs were conserved for both virulent and attenuated strains of MDPV.

Our determination of the genome sequence and construction of an infectious clone of FZ91-30 provide a foundation for further studies of MDPV in Muscovy ducklings.

\section{Conclusions}

The full-length genome sequence of strain FZ91-30 with 5131 nucleotides was determined. The amino acid mutations identified in the VP1 and Rep1 protein may contribute to the attenuation of FZ91-30 in Muscovy ducklings. Compared to the experimental procedure for viral rescue in cultured cells, plasmid transfection performed in embryonated goose eggs is a simple and efficacious method for rescue of infectious MDPV. Generation of the infectious plasmid pFZ may facilitate future studies of MDPV virulence.

\section{Methods}

Virus propagation and purification

FZ91-30 was diluted 1:50 in sterile saline containing penicillin $(2,000 \mathrm{U} / \mathrm{ml})$ and streptomycin $(2,000 \mathrm{U} / \mathrm{ml})$ and inoculated into the chorioallantoic cavity of 11day-old embryonated goose eggs. Embryos died from MDPV infection after $48 \mathrm{~h}$ were kept at $4{ }^{\circ} \mathrm{C}$ for $6 \mathrm{~h}$, and then their allantoic fluid was pooled. Approximately $300 \mathrm{ml}$ of allantoic fluid was centrifuged at 11, $000 \mathrm{~g}$ for $20 \mathrm{~min}$. Chloroform $(1 / 2 v / v)$ was added to the supernatant, shaken intensively, and then subjected to centrifugation at $11,000 \mathrm{~g}$ for $20 \mathrm{~min}$. The upper aqueous phase containing virus was transferred and pelleted by ultracentrifugation at 150,000 $\mathrm{g}$ for $3 \mathrm{~h}$ (SW32Ti rotor, Beckman), and the virus-containing pellet was resuspended in $5 \mathrm{ml}$ of TE buffer $(50 \mathrm{mM}$ Tris, 20 mM EDTA, pH 8.0).

\section{Viral DNA extraction}

Viral DNA was extracted as previously described [26], dissolved in STE buffer (10 mM Tris, $1 \mathrm{mM}$ EDTA, $100 \mathrm{mM} \mathrm{NaCl}, \mathrm{pH} 8.0$ ), and then annealed by heating to $95{ }^{\circ} \mathrm{C}$ for $5 \mathrm{~min}$ followed by slow cooling to $60{ }^{\circ} \mathrm{C}$.

\section{Construction of the infectious clone}

To facilitate cloning, the EcoRV site was replaced with NcoI (New England Biolabs, Ipswich, USA) in plasmid pBluescript II SK (Agilent, Santa Clara, USA), resulting in plasmid pBSKN. MDPV dsDNA was digested with NcoI, which cuts the genome at nucleotide position 723 . The digested fragments were separated using electrophoresis and purified with a gel extraction kit (Tiangen, Peiking, China). The 0.7-kb fragment was ligated into the HincII-NcoI site of pBSKN, generating plasmid pBSKNL. The 4.4-kb fragment was ligated into the NcoISmaI site of pBSKN, generating plasmid pBSKNR (Fig. 3a). The ligated products were transformed into competent cells of the Sure strain \{e14-(McrA-), $\Delta$ (mcrCB-hsdSMRmrr) 171 endA1 gyrA96 thi-1 supE44 relA1 lac recB recJ sbcC umuC::Tn5 (Kanr) uvrC [F' proAB lacIqZ $\Delta M 15$ Tn10 (Tetr)]\} (Agilent, Santa Clara, USA). pBSKNL was digested with $\mathrm{XhoI}$ and $\mathrm{NcoI}$, thus generating a $0.7-\mathrm{kb}$ fragment with XhoI/NcoI sites at either end. The 0.7-kb fragment was then ligated into $X h o \mathrm{I} / \mathrm{NcoI}$-digested pBSKNR, resulting in plasmid pFZ that contained the entire genome of the FZ91-30 strain.

\section{Sequence analysis and comparison}

The cloned genomic fragments were sequenced using an ABI-PRISM3730 automated sequencer and BigDye terminators v3.1 (Applied Biosystems, Foster City, USA). To overcome the difficulties in sequencing the ITRs, the plasmids were digested with SphI and BamHI or with XhoI. SphI cut the ITR in the middle loop position and the resulting fragments were sub-cloned into pUC19 or pBluescript II for sequencing. The sequences were assembled using SeqManII software included in the Lasergene package 5.0 (DNASTAR, Madison, USA). The complete genome sequence was submitted to GenBank, accession number KT865605.

For comparative studies, the complete MDPV genome sequences of strain FM and YY and the partial genome sequences of five pathogenic strains of MDPV (Table 1) were retrieved from GenBank. The hosts for three Taiwanese isolates (90-0215, 90-0219, and 97-0104) were Muscovy duck, mule duck, and goose, respectively [27]. 89384 was a France isolate from Muscovy duck [28]. P strain was isolated from Muscovy duck in mainland of China [29]. Sequence comparisons were performed using the MegAlign program packaged in the Lasergene package 5.0 . 
Table 1 MDPV isolates used in this study for sequence comparison

\begin{tabular}{|c|c|c|c|c|c|}
\hline Strain & Pathogenicity & Geographic origin & Genome region & Year of isolation & GenBank accession no \\
\hline FM & n. a. & Europe & Full-length & n. a. & U22967 \\
\hline 89384 & Pathogenic & France & VP1 & 1989 & Z68272 \\
\hline 90-0215 & Pathogenic & Taiwan & VP1 & 1990 & AY382891 \\
\hline 90-0219 & Pathogenic & Taiwan & VP1 & 1990 & AY382892 \\
\hline 97-0104 & Pathogenic & Taiwan & VP1 & 1997 & AY382893 \\
\hline$P$ & Pathogenic & China & Rep1; VP1 & 1988 & JF926697 \\
\hline YY & pathogenic & Chian & Full-length & 2000 & KX000918 \\
\hline FZ91-30 & Vaccine & China & Full-length & 1991 & KT865605 \\
\hline
\end{tabular}

n. a.: not available, but the isolation year is probably close to the 89384 strain

\section{Rescue of the infectious virus from the pFZ plasmid}

The pFZ plasmid was premixed with Lipofectamine 2000 (Invitrogen, Carlsbad, USA) at a ratio of 1:2.5 ( $\mu \mathrm{g}: \mu \mathrm{l})$. The transfection mixture was injected into the chorioallantoic membranes of 11-day-old embryonated goose eggs $(0.25 \mathrm{ml}$ per egg, $2.0 \mu \mathrm{g}$ of plasmid). Simultaneously, transfection of the vector plasmid pBSKN was performed as control.

When the embryos died from viral infection after $48 \mathrm{~h}$ post-transfection, their allantoic fluid was pooled. The allantoic fluid was 1:50 diluted in sterile saline and passaged in 11-day-old embryonated goose eggs four times. The presence of the rescued virions was detected by PCR using a pair of primers (5'-GGAACAAACCCA GACTCAAA-3' and 5'-CCAATCAGCCTATCTTCTA CAT-3') targeting the MDPV VP1 gene. The genome of the rescued virus was further amplified by using the PrimeSTAR Max DNA polymerase (Takara, Dalian, China) after PCR amplification of overlapping viral genome fragments. All amplified fragments were gelpurified and submitted to direct sequencing.

\section{Introduction of a genetic marker into the rescued virus}

To exclude the possibility that the rescued virus originated from contamination during the course of transfection or passaging, a single nucleotide mutation $(\mathrm{C} \rightarrow \mathrm{T})$ was introduced into the Rep1 gene in the pFZ plasmid by overlap PCR. The synonymous nucleotide mutation results in loss of the NdeI restriction endonuclease site.

To realize the purpose, two pairs of primers used in the overlap PCR were designed and listed (Table 2). The

Table 2 Primers used in the overlap PCR to introduce a nucleotide mutation to the rescued virus

\begin{tabular}{|c|c|c|}
\hline Primer name & Primer sequence $^{a}$ & Amplification size \\
\hline PM-1 & 5' TCTCCCCATGGTTACTCTGG 3' & \multirow{2}{*}{$839 \mathrm{bp}$} \\
\hline PM-2 & 5' GTCCGTAGAGCCATATAGGCAT 3' & \\
\hline PM-3 & 5' ATGCIATATGGCTCTACGGAC 3' & \multirow{2}{*}{$657 \mathrm{bp}$} \\
\hline PM-4 & 5' TCTCAGGAGATCTCTCTCCGGACT 3' & \\
\hline
\end{tabular}

${ }^{a}$ The restriction enzyme site ( $\mathrm{Ncol}$ and $\mathrm{Bg} / \mathrm{Il}$ ) in the primers was denoted with italics and the mutated nucleotide set in primers was underlined upstream primer PM-1 and the downstream PM-2 were used to amplify an 839-bp fragment, and the upstream primer PM-3 and the downstream PM-4 were used to amplify a 657-bp fragment. One ng of pFZ plasmid was used as the DNA template in $50 \mu$ l of PCR reaction mixture. The amplified fragments were purified with a gel recovery kit (Tiangen, Peiking, China) and dissolved in $30 \mu \mathrm{l}$ of deionized water. The recovered DNA fragments ( $1 \mu \mathrm{l}$ of each fragment) were used as the DNA templates in the subsequent overlap PCR. For overlap PCR, $50 \mu \mathrm{l}$ of reaction volume also included $25 \mu \mathrm{l}$ of primeSTAR Max Premix (Takara, Japan), and 20 pmol of each primer (PM-1 and PM-4). The reaction condition was as follows: $95{ }^{\circ} \mathrm{C}$ for $1 \mathrm{~min}$ followed by 25 cycles of denaturation at $94{ }^{\circ} \mathrm{C}$ for $15 \mathrm{~s}$, annealing at $55^{\circ} \mathrm{C}$ for $25 \mathrm{~s}$, elongation at $72{ }^{\circ} \mathrm{C}$ for $23 \mathrm{~s}$, with a final extension step at $72{ }^{\circ} \mathrm{C}$ for $10 \mathrm{~min}$. The $1.5-\mathrm{kb}$ amplicon purified with the gel recovery kit (Tiangen, Peiking, China) was digested with $\mathrm{NcoI}$ and BglII and ligated with the linearized pFZ plasmid predigested with the same enzymes.

The engineered plasmid pFZ carrying the genetic marker was transfected into 11-day-old embryonated goose eggs to rescue the infectious virus as described above. A 1.5-kb DNA fragment containing the mutation site was amplified by PCR using primer PM-1 and PM-4 from the DNA template extracted from the rescued virions and digested with $\mathrm{NdeI}$.

\section{Biological Characterization of the rescued virus}

To study the titer and virulence of the rescued virus, the medium embryos lethal dose $\left(\mathrm{ELD}_{50}\right)$ was calculated by the method of Reed and Muench [30] and mean death time (MDT) was calculated by the method used to evaluate the virulence of Newcastle disease virus [31].

Briefly, the allantoic fluid was diluted in sterile saline to give a tenfold dilution series between $10^{-1}$ and $10^{-5}$. For each dilution, $0.2 \mathrm{ml}$ was inoculated into the allantoic cavity of each of five 11-day-old embryonated goose eggs, which were then incubated at $37{ }^{\circ} \mathrm{C}$. The remaining virus dilutions were retained at $4{ }^{\circ} \mathrm{C}$ and another five eggs were inoculated with $0.2 \mathrm{ml}$ of each 
dilution $8 \mathrm{~h}$ later and incubated at $37{ }^{\circ} \mathrm{C}$. Each egg was examined twice daily for 10 days and the times of any embryo deaths were recorded. The minimum lethal dose is the highest virus dilution that causes all the embryos inoculated with that dilution to die. MDT is the mean time in hours for the minimum lethal dose to kill all the inoculated embryos.

\section{Acknowledgement}

This work was supported by the National Natural Science Foundation of China (no. 31572551) and by a project funded by the Priority Academic Program Development of Jiangsu Higher Education Institutions.

\section{Authors' contributions}

JY W conceived and designed the experiments. JY W, Y H, and MX Z performed the experiments. PRH and GQ Z analyzed the data. JY W wrote the paper. PRH checked and revised the manuscript. All authors read and approved the final manuscript.

\section{Competing interests}

The authors declare that they have no competing interests.

\section{Ethics approval and consent to participate}

The procedure for inoculation of fertilized Muscovy duck eggs was approved by the Animal Care and Use Committee of Yangzhou University and performed in accordance with the "Guidelines for Experimental Animals" of the Ministry of Science and Technology (Beijing, China). No specific permissions were required for these locations/activities.

\section{Author details}

${ }^{1}$ College of Veterinary Medicine, Yangzhou University, 48 Wenhui East Road, 225009 Yangzhou, Peoples' Republic of China. ${ }^{2}$ College of Veterinary Medicine, Kansas State University, Manhattan, KS, USA.

Received: 13 April 2016 Accepted: 14 June 2016

Published online: 21 June 2016

References

1. Cotmore S, Agbandje-McKenna M, Chiorini JA, Mukha DV, Pintel DJ, Qiu JM, Soderlund-Venermo M, Tattersall P, Tijssen P, Gatherer D, Davisonet AJ. The family Parvoviridae. Arch Virol. 2014;159:1239-47.

2. Jestin V, Le Bras MO, Cherbonnel M, Le Gall G, Bennejean G. Mise en évidence de parvovirus (virus de la maladie de Derzsy) très pathogènes dans les élevages de canards de Barbarie. Rec Med Vet. 1991;167:849-57.

3. Fournier D, Gaudry D. Recent discoveries on waterfowl pathology: a new parvovirus of Muscovy ducks in France-Field vaccination trials. In: McNulty MS, McFerran JB, editors. Proceedings of the Commission of the European Communities Meeting on Virus Diseases of Poultry-New and Evolving Pathogens. Brussels: European Commission; 1992. p. 183-94.

4. Takehara K, Hyakutake K, Imamura T, Mutoh K, Yoshimura M. Isolation, identification and plaque titration of parvovirus from Muscovy ducks in Japan. Avian Dis. 1994;38:810-5.

5. Woolcock PR, Jestin V, Shivaprasad HL, Zwingelstein F, Arnauld C, McFarland MD, Pedersen JC, Senne DA. Evidence of Muscovy duck parvovirus in Muscovy ducklings in California. Vet Rec. 2000;146:68-72.

6. Chen YQ, Lin TL, Hu QL, Li YY, Zhou WM, Wu ZY. Isolation and identification of a Muscovy duckling parvovirus. Chi J Virol. 1993:9(3):228-34 (In Chinese).

7. Glávits R, Zolnai A, Szabó É, Ivanics É, Zarka P, Mató T, Palya V. Comparative pathological studies on domestic geese (Anser Anser Domestica) and Muscovy ducks (Cairina Moschata) experimentally infected with parvovirus strains of goose and muscovy duck origin. Acta Vet Hung. 2005;53:73-89.

8. Gall-Reculé GL, Jestin V. Biochemical and genomic characterization of Muscovy duck parvovirus. Arch Virol. 1994;139:121-31.

9. Zadori Z, Stefancsik R, Rauch T, Kisary J. Analysis of the complete nucleotide sequences of goose and muscovy duck parvoviruses indicates common ancestral origin with adeno-associated virus 2. Virology. 1995;212:562-73.

10. Wang XS, Ponnazhagan S, Srivastava A. Rescue and replication of adenoassociated virus type 2 as well as vector DNA sequences from recombinant plasmids containing deletions in the viral inverted terminal repeats: selective encapsidation of viral genomes in progeny virions. J Virol. 1996;70:1668-77.

11. Qiu J, Cheng F, Yoto Y, Zadori Z, Pintel D. The expression strategy of Goose parvovirus exhibits features of both the Dependovirus and Parvovirus genera. J Virol. 2005;79:11035-44.

12. Li L, Qiu J, Pintel DJ. The choice of translation initiation site of the rep proteins from goose parvovirus P9-generated mRNA is governed by splicing and the nature of the excised intron. J Virol. 2009;83:10264-8.

13. Snyder RO, Im DS, Muzyczka N. Evidence for covalent attachment of the adeno-associated virus (AAV) Rep protein to the ends of the AAV genome. IVirol. 1990:64:6204-13.

14. Legendre $\mathrm{D}$, Rommelaere J. Targeting of promoters for trans activation by a carboxy-terminal domain of the NS-1 protein of the parvovirus minute virus of mice. J Virol. 1994;68:7974-85.

15. Gough RE, Spackman D. Studies with a duck embryo adapted goose parvovirus vaccine. Avian Pathol. 1982;11:3503-10.

16. Chen YQ, Hu QL, Li YY, Chen SY. Selection of attenuated vaccine strain of Muscovy duckling parvovirus and its biological characteristics. Chin J Vet Sci. 1996;16(2):118-21 (In Chinese).

17. Maurin-Bernaud L, Goutebroze S, Merdy O, Chanay A, Cozette V, Le Gros FX. Efficacy of a new attenuated duck parvovirosis vaccine in Muscovy ducks. Vet Rec. 2014;175(11):281.

18. Meng SS, Wang YK. Study of some properties of Muscovy duck parvovirus. J Jiangsu Agric Coll. 1994;15(4):52-7 (In Chinese).

19. Zhi N, Zadori Z, Brown KE, Tijssenet P. Construction and sequencing of an infectious clone of the human parvovirus B19. Virology. 2004;318:142-52.

20. Lee KH, Kim YG, Jo EC. Shuttle PCR-based cloning of the infectious adenoassociated virus type 5 genome. J Virol Methods. 2003;111:75-84.

21. Samulski RJ, Srivastava A, Berns Kl. Rescue of adeno-associated virus from recombinant plasmids: gene correction within the terminal repeats of AAV. Cell. 1983:33:135-43.

22. Parker JSL, Murphy WJ, Wang D, O'Brien SJ, Parrish CR. Canine and feline parvoviruses can use human or feline transferrin receptors to bind, enter, and infect cells. J Virol. 2005;75:3896-902.

23. Yin XC, Zhang SM, Gao YL, Li JZ, Tan SY, Liu HY, Wu XY, Chen YH, Liu M, Zhang Y. Characterization of monoclonal antibodies against waterfowl parvoviruses VP3 protein. Virol J. 2012:9:288

24. Zadori Z, Szelei J, Lacoste MC, Li Y, Gariepy S, Raymond P, Allaire M, Nabi IR, Tijssen P. A viral phospholipase A2 is required for parvovirus infectivity. Dev Cell. 2001;1:291-302.

25. Boisvert M, Bouchard-Lévesque V, Fernandes S, Tijssen P. Classic nuclear localization signals and a novel nuclear localization motif are required for nuclear transport of porcine parvovirus capsid proteins. J Virol. 2014;88(20):11748-59.

26. Bossis I, Chiorini JA. Cloning of an avian adeno-associated virus (AAAV) and generation of recombinant AAAV particles. J Virol. 2003;77:6799-810.

27. Tsai HJ, Tseng $\mathrm{CH}$, Chang PC, Mei K, Wang SC. Genetic variation of viral protein 1 genes of field strains of waterfowl parvoviruses and their attenuated derivatives. Avian Dis. 2004:48:512-21.

28. Gall-Reculé $G L$, Jestin V, Chagnaud $P$, Blanchard $P$, Jestin A. Expression of muscovy duck parvovirus capsid proteins (VP2 and VP3) in a baculovivurs expression system and demonstration of immunity induced by the recombinant proteins. J Gen Virol. 1996;77:2159-63.

29. Wang S, Cheng XX, Chen SY, Zhu XL, Chen SL, Lin FQ, Li ZL. Genetic characterization of a potentially novel goose parvovirus circulating in Muscovy duck flocks in Fujian Province, China. J Vet Med Sci. 2013;75:1127-30.

30. Reed $\amalg$, Müench $H$. A simple method for estimating fifty percent endpoints Am J Hyg. 1938:27:493-7.

31. Pearson JE, Senne DA, Alexander DJ, Taylor WD, Peterson LA, Russell PH. Characterization of Newcastle disease virus (avian paramyxovirus-1) isolated from pigeons. Avian Dis. 1987;31:105-11. 